Neurosurg Focus 19 (2):E6, 2005

\title{
Transpetrosal approaches to the posterior fossa
}

\author{
Ramachandra P. Tummala, M.D., Ernesto Coscarella, M.D., \\ AND JACQUES J. Morcos, M.D., F.R.C.S. \\ Department of Neurological Surgery, University of Miami School of Medicine, Miami, Florida
}

\begin{abstract}
Resection of the petrous temporal bone to various degrees provides different levels of access to lesions of the posterior fossa. Although their nomenclature can be confusing, the numerous variants of the transpetrosal approaches can be classified broadly into anterior and posterior groups. The posterior transpetrosal approaches include the retrolabyrinthine, translabyrinthine, and transcochlear, whereas the ones in the anterior group are extensions of the basic middle fossa approach. Both the anterior and posterior approaches have the potential of exposing the cerebellopontine angle and the petroclival region.

The posterior approaches are based on the standard mastoidectomy and involve resection of the petrous bone to various degrees. This results in progressively increased exposure anteriorly, but comes at the expense of hearing in the translabyrinthine approach and of hearing and facial strength in the transcochlear approach.

In contrast, the middle fossa approaches spare the lateral petrous bone and involve resection of the medial petrous bone to various degrees. All of the middle fossa approaches are designed to preserve hearing. Extensions of the middle fossa approaches involve resection of bone within the Kawase rhomboid and division of the tentorium to provide exposure of the posterior fossa.
\end{abstract}

\section{KEY WoRDS • petrosal approach • Kawase approach • presigmoid region • retrolabyrinthine approach • translabyrinthine approach - transcochlear approach}

Resection of the petrous temporal bone to various degrees has overcome the limitations associated with traditional exposures of the posterior fossa. The combination of transpetrosal procedures with other approaches has resulted in many options for obtaining wide surgical exposure of the petroclival region. Nevertheless, the improved access to the posterior fossa through the transpetrosal route comes at the price of increased morbidity. The variations of the transpetrosal approaches are numerous, and the resulting nomenclature can be quite confusing. Frequently, these variations overlap with one another and have minor distinguishing nuances. All of these approaches have been categorized under the broad terms of anterior and posterior transpetrosal approaches. ${ }^{11}$ For our purposes, we shall follow this convention and focus on the surgical anatomy and technical points of the major posterior and anterior approaches. We generally perform these operations in collaboration with our neurootology colleagues.

\section{POSTERIOR TRANSPETROSAL APPROACHES}

The posterior temporal bone dissection includes the retrolabyrinthine, translabyrinthine, and transcochlear approaches. All three of these are extensions of the basic mastoidectomy. The retrolabyrinthine approach is the most minimal of the three, whereas the other two approaches represent a graduated increase in the extent of petrous bone

Abbreviations used in this paper: $\mathrm{BA}=$ basilar artery; $\mathrm{CPA}=$ cerebellopontine angle; EAC $=$ external auditory canal; GSPN = greater superficial petrosal nerve; IAC = internal auditory canal; $\mathrm{ICA}=$ internal carotid artery; $\mathrm{MR}=$ magnetic resonance; $\mathrm{SSC}=$ superior semicircular canal. resection (Fig. 1). The increased bone resection involved in the translabyrinthine and transcochlear approaches results in a corresponding increase in anterior exposure. Nevertheless, the progressive gain in exposure is obtained at the expense of a risk to seventh and eighth cranial nerve function.

\section{Retrolabyrinthine Approach}

We routinely place the patient supine with the ipsilateral shoulder elevated and the head turned to the opposite side so that the mastoid process is at the highest point in the field. We do not hesitate to use the lateral position for patients with large or inflexible necks. The abdomen is also draped in preparation for an autologous fat graft harvest. A retroauricular $\mathrm{C}$-shaped incision is made, and the scalp flap is retracted anteriorly. If a combined approach with a subtemporal craniotomy is planned, as is most common, the incision is curved anteriorly to expose the root of the zygoma just anterior to the tragus (Fig. 2). The translabyrinthine and transcochlear approaches are dependent on and are progressions of the retrolabyrinthine exposure.

The mastoidectomy is initiated by wide removal of the cortical bone overlying the mastoid. Detailed descriptions of this procedure are found elsewhere. ${ }^{12}$ The anterior boundary of the cortical bone removal is the posterior wall of the EAC. The remaining boundaries are the temporal line (linea temporalis or supramastoidal crest) superiorly and the tip of the mastoid inferiorly. The sigmoid sinus is skeletonized inferiorly through the infralabyrinthine air cells to the jugular bulb.

After exposure of the sinodural angle between the middle fossa dura, the superior petrosal sinus, and the sigmoid sinus, the next task is identification of the mastoid antrum. 


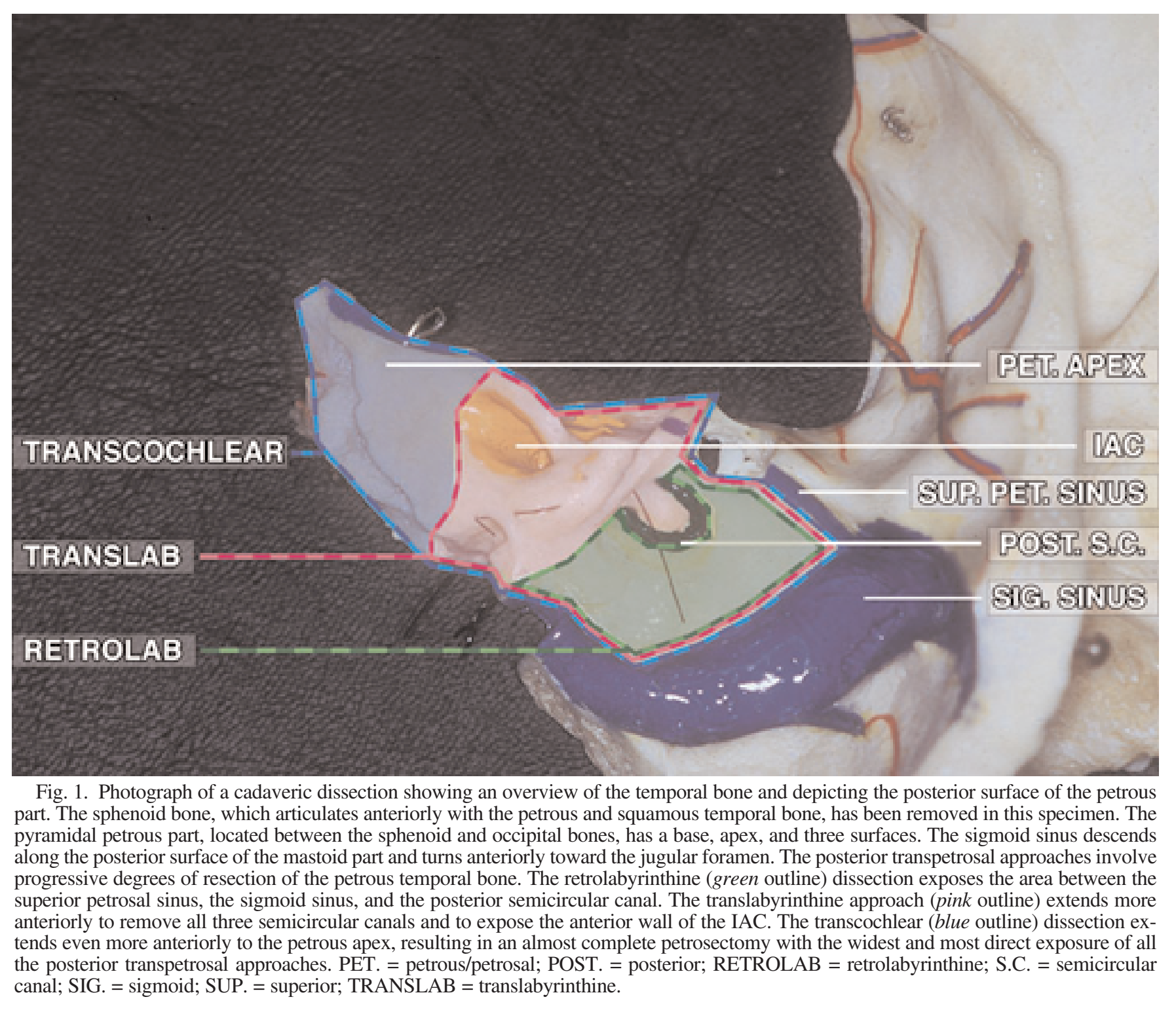

Following the middle fossa dural plate and posterior EAC wall deep into the Macewen triangle leads to the antrum. The short process of the incus can now be identified in the fossa incudis and helps localize the lateral semicircular canal, which lies immediately below the antrum. The external genu of the facial nerve is located at the inferior surface of the lateral semicircular canal. Removal of air cells inferiorly also exposes the digastric ridge, and removal of the remaining air cells posterior to the lateral semicircular canal exposes the crossing posterior semicircular canal. Removal of the remaining inferior mastoid and retrofacial air cells skeletonizes the vertical segment of the facial nerve, which is located within the fallopian canal anterior to the digastric ridge. Complete skeletonization is unnecessary, and a layer of cortical bone is left intact over the facial nerve to prevent injury. Drilling through the supralabyrinthine air cells and following the sinodural angle to the petrous ridge will lead to the SSC. All three semicircular canals are therefore skeletonized but preserved. Removal of the bone plates on the middle and posterior fossa dura mater and the sigmoid sinus completes the extradural part of the retrolabyrinthine approach (Fig. 3). This approach provides exposure of the CPA but does not give the surgeon adequate visualization of the anterior brainstem or petroclival region (Fig. 4). Adequate exposure of the IAC is possible in most cases. ${ }^{1}$ Visualization of the CPA may be compromised if the sigmoid sinus is larger than normal or anteriorly displaced.

\section{Translabyrinthine Approach}

This dissection is an anterior extension of the retrolabyrinthine approach (Fig. 5). The initial part of the translabyrinthine dissection includes all of the steps outlined for the retrolabyrinthine approach. The limits of the dissection are the mastoid segment of the facial nerve anteriorly, the jugular bulb inferiorly, and the middle fossa superiorly. ${ }^{2}$

In the translabyrinthine approach, the semicircular ca- 


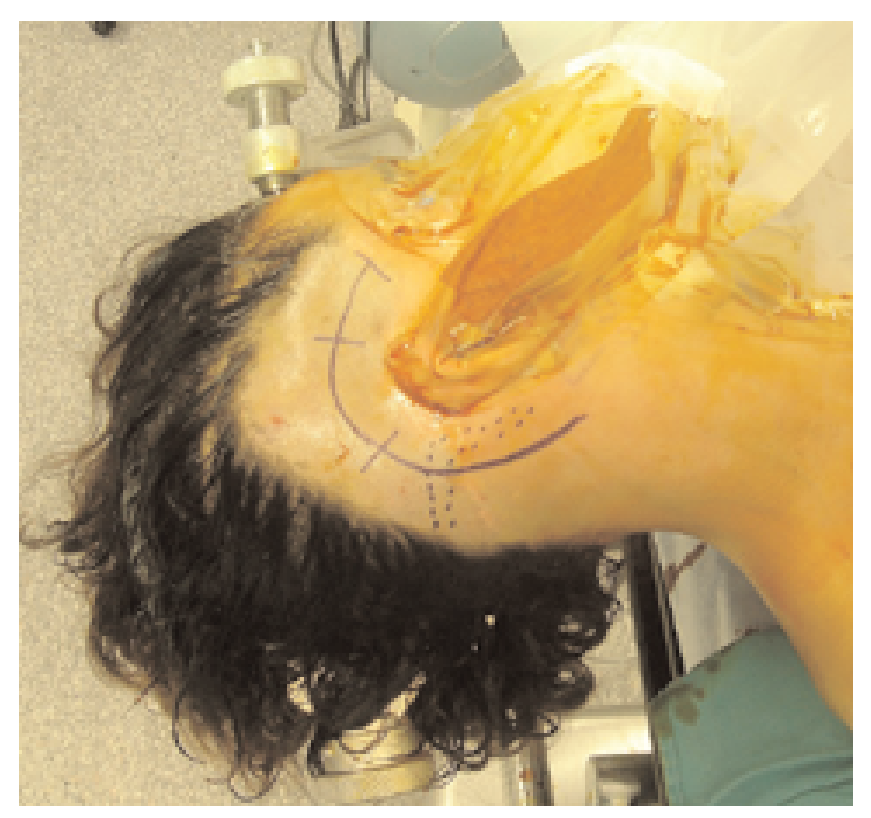

Fig. 2. Intraoperative photograph showing the standard retroauricular incision for a posterior transpetrosal combined subtemporal approach. The incision is curved anteriorly to allow exposure of the root of the zygoma just anterior to the tragus. The presumed locations of the transverse and sigmoid sinuses (dashed lines), based on the surface anatomy, are also indicated.

nals are removed and the vestibule is opened to reveal the nerves to the lateral, inferior, and superior ampullae. The superior ampulla represents the lateral wall of the IAC fundus, and additional bone is removed until the IAC is skeletonized. The superior vestibular nerve is encountered first when the IAC is opened; it lies posterosuperiorly within the canal and is separated from the facial nerve by the thin vertical crest (the Bill bar). One advantage of the translabyrinthine approach is that the vestibular nerves are encountered before the facial nerve; this decreases the risk of injury to the facial nerve. Removal of bone from the superior wall of the IAC must be done cautiously because the facial nerve lies superficially under the dura mater and can be injured. The result of the additional bone resection in the translabyrinthine exposure is increased access to the anterolateral brainstem and inferior clivus (Fig. 6). Nevertheless, resection of the labyrinth and sacrifice of hearing are necessary to produce this increased anterior access.

The partial translabyrinthine approach is a valuable variant. It represents an attempt at combining improved exposure of the petroclival junction compared with the retrolabyrinthine approach, without the inevitable deafness incurred by the full translabyrinthine approach. It requires for its success a meticulous drilling of either the superior or posterior semicircular canal (depending on whether the upper or the lower clivus, respectively, needs to be exposed), with special precautions taken to minimize loss of endolymph. Hearing can indeed be preserved in most instances.

\section{Transcochlear Approach}

This dissection, in turn, is an anterior extension of the translabyrinthine approach. The transcochlear approach ne- cessitates resection of the cochlea, division of the GSPN, and posterior transposition of the facial nerve. In the original description, the anterior limits of this dissection were the posterior wall of the EAC and the middle ear. ${ }^{5}$ Variations of this procedure, however, routinely require resection of the middle ear. The initial part of the dissection is the same as for the translabyrinthine approach, except that the EAC is transected, and the skin of the cartilaginous canal is sutured closed. The facial nerve is skeletonized from its entrance into the IAC to the stylomastoid foramen. The facial recess, an area of air cells contained between the vertical facial nerve medially, the chorda tympani laterally, and the fossa incudis superiorly, is opened to enter the middle ear. In the modified transcochlear approach, the middle ear ossicles are removed. Both the chorda tympani and the GSPN are sectioned at their origins from the facial nerve. These maneuvers free the descending segment of the facial nerve and allow its transposition posteriorly.

Next, the cochlea is resected, starting at the basal turn and proceeding anteriorly to the petrous ICA. The septum between the basal turn and the ICA is also resected along with the carotid ridge, which is a thin plate of bone separating the ICA from the jugular bulb. This maneuver exposes the jugular bulb completely. Therefore, the surgeon must be aware of the ninth, 10th, and 11th cranial nerves as they exit the jugular foramen.

Completion of the transcochlear approach as described earlier results in removal of the posterior petrous and tympanic temporal bone (Fig. 7). The resulting wide exposure creates direct views of the clivus, primarily its middle half, the anterior brainstem, and the BA (Fig. 8). Extensions of this approach through the middle fossa can be used to access lesions of the infratemporal fossa and posterior nasopharynx.

A rarely used variant of the transcochlear approach is the transotic approach. In it, the facial nerve is not transposed and tumor resection is performed in front of and behind the undisturbed facial nerve.

Closure of the posterior transpetrosal approach involves meticulous inspection and obliteration of potential cerebrospinal fluid fistulas. Any remaining air cells are sealed with bone wax. The middle ear and IAC are filled with autologous muscle or fat grafts. The cavity created by the mastoidectomy is filled with autologous abdominal fat, and the wound is closed in layers, including a rotation flap of the temporalis fascia, with application of a pressure dressing at the end of the procedure.

\section{ANTERIOR TRANSPETROSAL APPROACHES}

\section{Middle Fossa Approach}

The middle fossa approach by itself does not provide meaningful access to the posterior fossa. Nevertheless, it lays the foundation for extensions that expose the anterior CPA and petroclival region. Unlike the posterior transpetrosal approaches, the middle fossa dissection spares the lateral petrous bone and involves the removal of the medial part only. The middle fossa route provides access to the petrous apex and small lesions within the IAC. We place the patient supine with the ipsilateral shoulder elevated and the head turned to the opposite side. The neck is slightly extended, with the vertex of the head directed toward the 

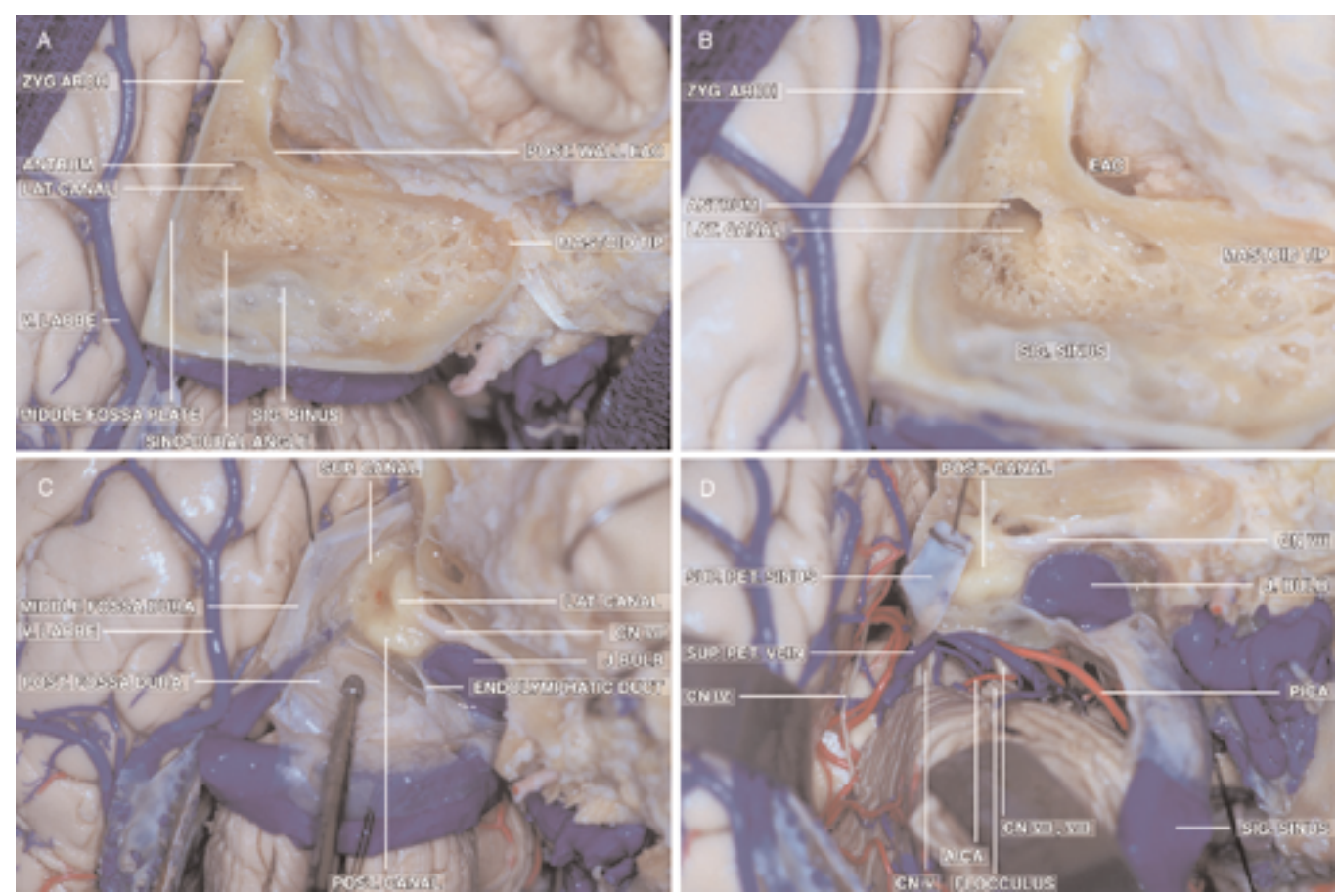

Fig. 3. Photographs of a cadaveric dissection showing the retrolabyrinthine approach, right side. This dissection was done in the context of a combined presigmoid subtemporal approach. A basic mastoidectomy was the fundamental step in this dissection. A: The boundaries of the cortical bone removal are the posterior wall of the EAC anteriorly, the middle fossa dural plate superiorly, and the sigmoid sinus posteriorly. B: The mastoid antrum lies deep to the suprameatal or Macewen triangle, which is bound superiorly by the posterior root of the zygomatic arch, posteriorly by a vertical line extending along the posterior border of the external auditory meatus, and inferiorly by the superior margin of the external auditory meatus. C: The sigmoid and superior petrosal sinuses, the middle fossa dura, and the posterior fossa dura have been skeletonized to reveal the sinodural angle. The bone labyrinth has been revealed without violating the individual semicircular canals. D: Overview of the retrolabyrinthine approach after dural opening, showing the limited exposure of the CPA and brainstem. $\mathrm{AICA}=$ anterior inferior cerebellar artery; $\mathrm{CN}=$ cranial nerve; $\mathrm{J} .=$ jugular; LAT.$=$ lateral; PICA = posterior inferior cerebellar artery; $\mathrm{V}$. = vein; ZYG. = zygomatic.
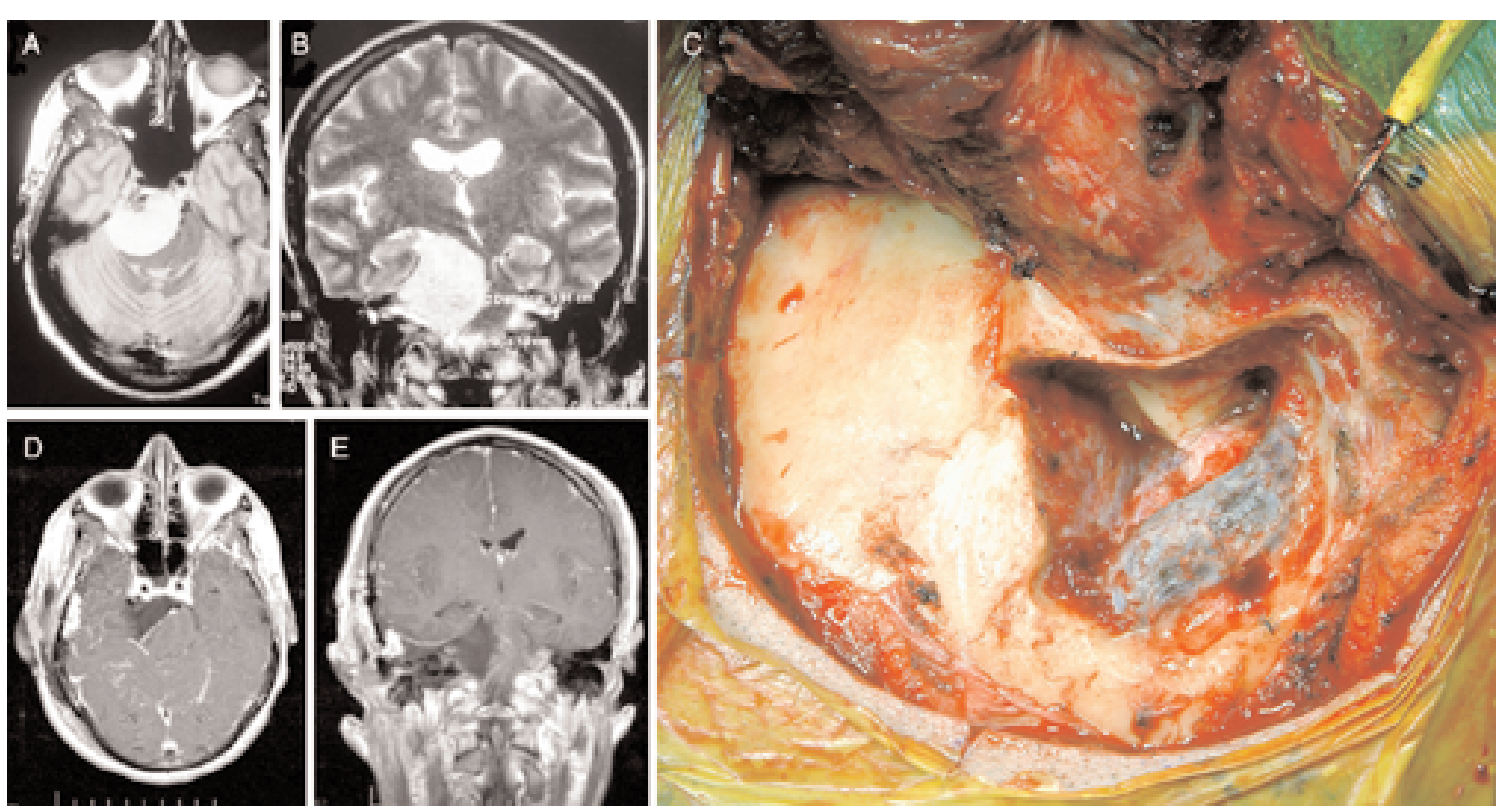

Fig. 4. A and B: Axial and coronal $\mathrm{T}_{2}$-weighted MR images obtained in a 50-year-old woman presenting with a 1-year history of headaches, dysarthria, and imbalance. Her neurological examination revealed normal hearing and mild right dysmetria. To preserve hearing, this large petroclival meningioma was resected via a combined retrolabyrinthine-subtemporal approach. C: Intraoperative view obtained after completion of the extradural portion of the procedure. The middle and posterior fossa dural plates and the sigmoid sinus have been skeletonized. Note the limited but adequate working space between the sigmoid sinus and the bone labyrinth, although it averages $1 \mathrm{~cm}$. $\mathrm{D}$ and E: Contrast-enhanced $\mathrm{T}_{1}$-weighted axial and coronal MR images demonstrating gross-total resection. Postoperatively, the patient experienced a right sixth cranial nerve palsy and transient worsening of her right-sided dysmetria. Her facial strength and hearing remained intact. 

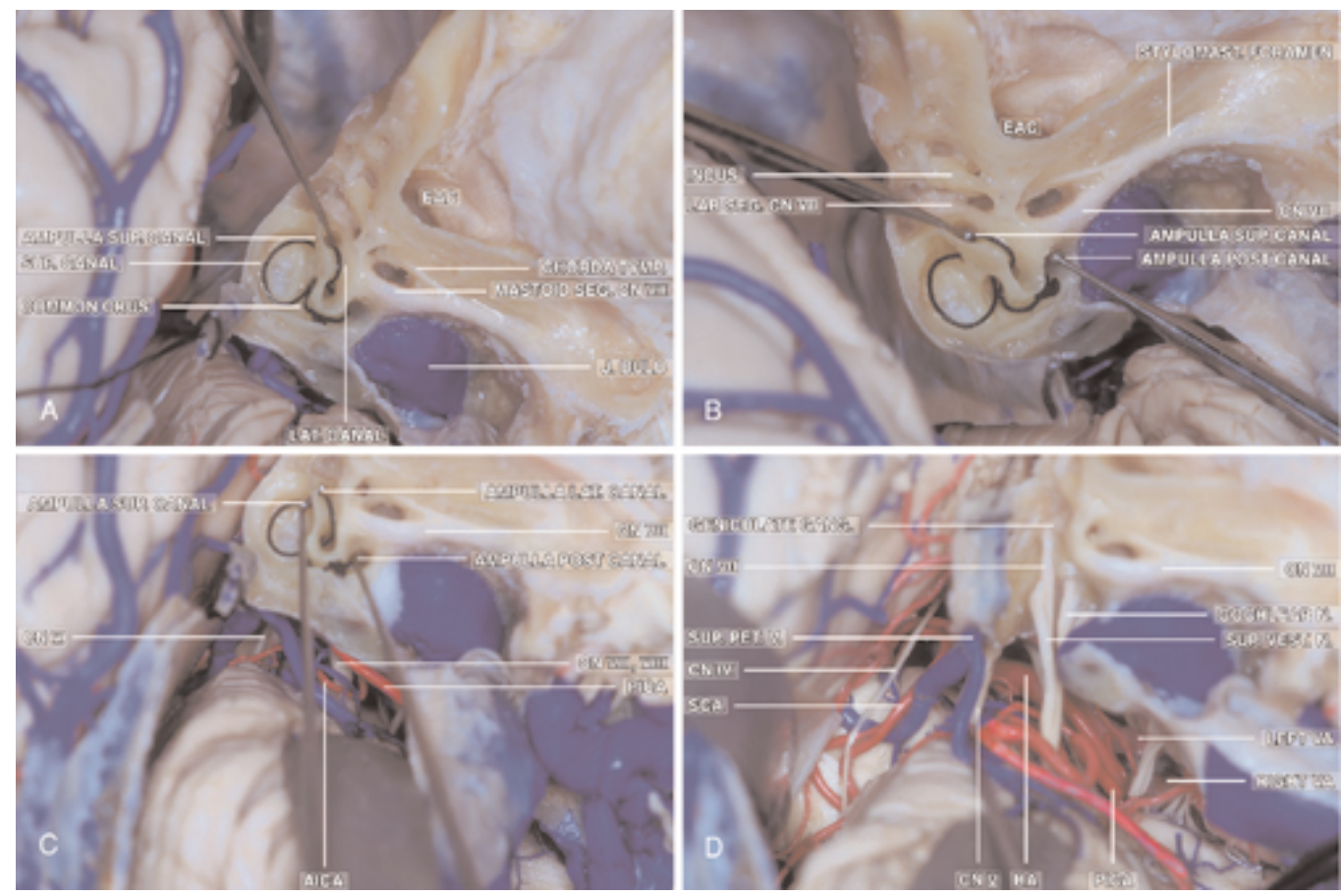

Fig. 5. Photographs of cadaveric dissections showing the translabyrinthine approach, right side. A: The bone labyrinth has been opened to expose the individual semicircular canals. The tip of the instrument is in the ampulla of the SSC, which lies directly beneath the arcuate eminence. The lateral semicircular canal is the most laterally projecting one and the first one encountered in the translabyrinthine approach. It is the landmark for identifying the underlying labyrinthine segment of the facial nerve. B: Magnified view. The two instruments lie within the ampullae of the superior and posterior semicircular canals. These landmarks represent the superior and inferior aspects of the deep lateral portion of the IAC, respectively. C: Orientation of the IAC in relation to the seventh and eighth cranial nerve complex. D: Overview of the translabyrinthine dissection showing a wider exposure of the anterior CPA and prepontine cistern. In this specimen the exposure is relatively limited because of a high jugular bulb. GANG. = ganglion; LAB = labyrinthine; N. = nerve; SEG. = segment; STYLOMAST. = stylomastoid; TYMP. = tympan; VEST. = vestibular.
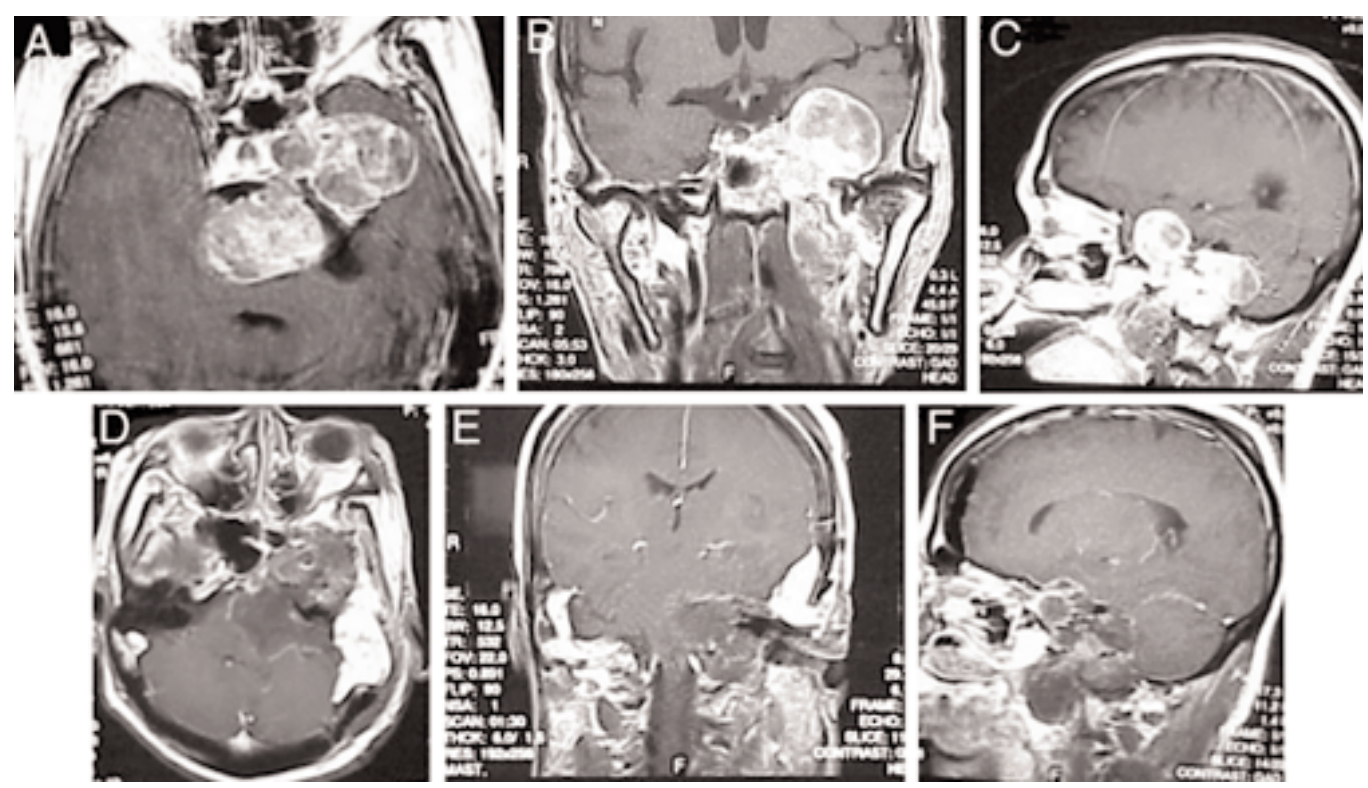

Fig. 6. This 67-year-old woman presented with a 4-year progression of left hemiparesis, hoarseness, and left facial palsy (House-Brackmann Grade III). She had become increasingly lethargic in the days prior to her presentation. Her disease had been inappropriately labeled as a stroke based on her computerized tomography scan. An audiogram confirmed absence of hearing, and laryngoscopy showed left vocal cord paresis. A-C: Contrast-enhanced $\mathrm{T}_{1}$-weighted axial (A), coronal (B), and sagittal (C) MR images revealing a heterogeneously enhancing mass occupying the left middle and posterior fossae and causing severe compression of the brainstem. The mass appears to invade the cavernous sinus as well as extending through the foramen ovale and the jugular foramen. This chondrosarcoma was resected and the brainstem was decompressed via a combined translabyrinthine subtemporal approach. D-F: Postoperative contrasted axial (D), coronal (E), and sagittal (F) views confirm complete resection and show the abdominal fat graft extradurally. Gratifyingly, the patient's mobility improved after surgery. 

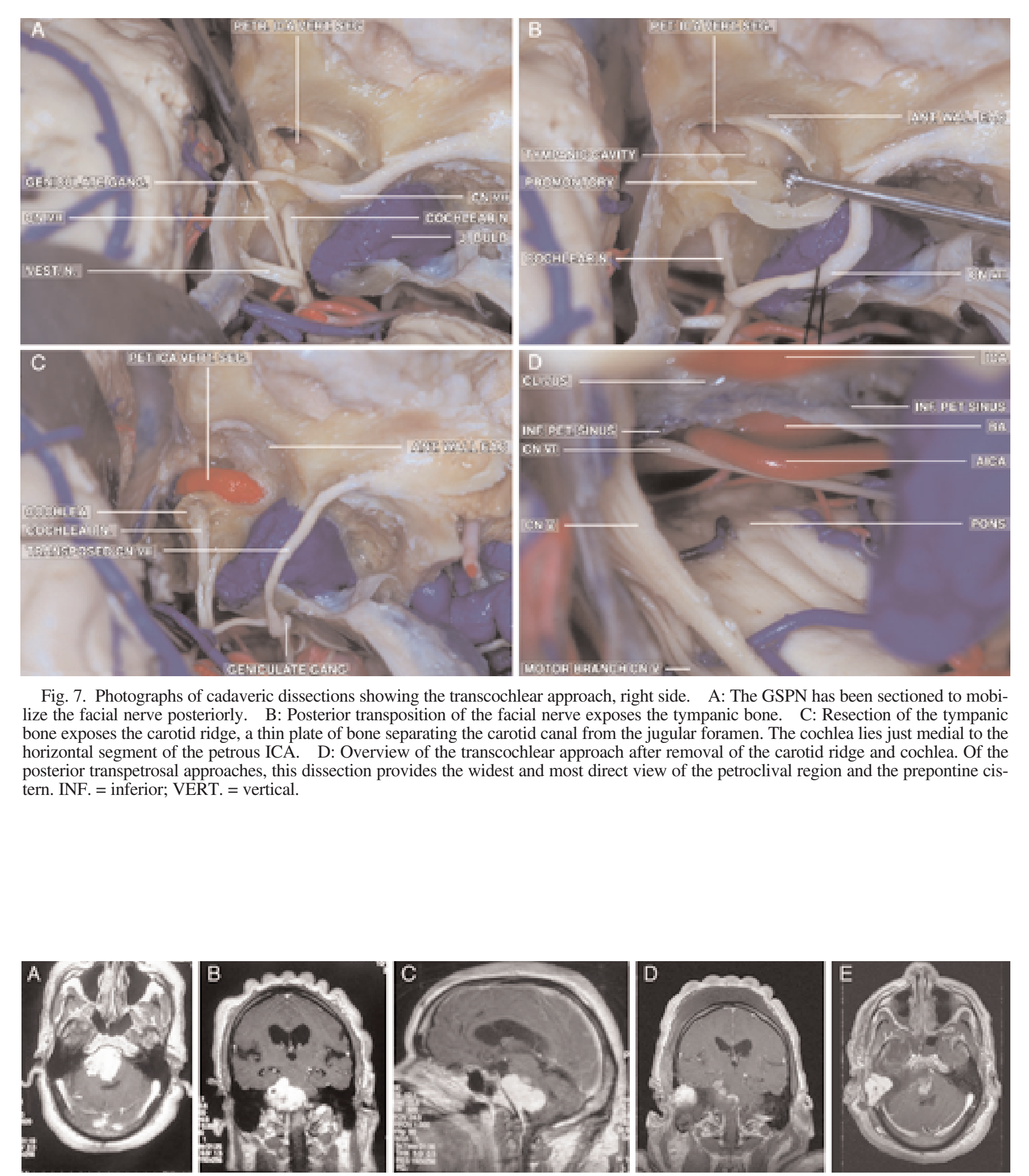

Fig. 8. This 60-year-old man had undergone radiation therapy without surgery at another facility 4 years earlier for a petroclival meningioma. He presented to us with very poor balance, dysphagia, complete hearing loss, and severe facial weakness on the right side. A-C: Contrast-enhanced $\mathrm{T}_{1}$-weighted axial (A), coronal (B), and sagittal (C) MR images revealing that the enhancing lesion had grown significantly in 4 years. These images (as well as the $\mathrm{T}_{2}$-weighted sequences that are not shown) predicted a poor dissection plane between the tumor and the brainstem. Because of the hearing loss and nearly complete facial palsy, we resected this lesion subtotally via a combined transcochlear-subtemporal approach. D and E: The postoperative MR images demonstrate a small amount of tumor that was left attached to the brainstem, which is markedly decompressed. 
floor. Placement of a lumbar drain is essential to relax the brain and elevate the temporal lobe safely. We use a straight scalp incision that begins just anterior to the tragus at the level of the zygoma and ends above the superior temporal line. A temporal craniotomy with two-thirds anterior to and one-third posterior to the EAC is then completed. The remaining squamous temporal bone is drilled down to the level of the floor of the middle fossa. A self-retaining retractor keeps the temporal lobe elevated during the bone drilling.

Before drilling any bone, several landmarks must be identified for orientation of the regional anatomy. Individual variations in anatomy must also be recognized. The temporal lobe is elevated extradurally, and the first landmark is the middle meningeal artery as it emerges from the foramen spinosum. After division of this vessel and further elevation of the dura mater, the next landmark is the arcuate eminence, a round elevation of bone overlying the SSC (the arcuate eminence may be absent in $15 \%$ of patients). ${ }^{7}$ The structures encountered next are the lesser (lateral) and greater (medial) superficial petrosal nerves. The dura mater is lifted gently in a posterior-to-anterior direction to avoid excessive traction on the facial nerve. We try to preserve the GSPN whenever possible to avoid postoperative "dry eye" from impaired lacrimation. In rare cases, sacrifice of this structure may be necessary to prevent traction injury to the geniculate ganglion. The GSPN marks the lateral margin of the underlying horizontal segment of the petrous ICA. The floor of the middle fossa may be quite thin or even absent at this location, and the ICA may be injured easily (Fig. 9).

The goal of the middle fossa approach is the identification of the IAC in the floor of the middle fossa. Although identification of this structure via the aforementioned approach may seem like a straightforward exercise, small errors can lead to injury of the seventh and eighth cranial nerves. Once the landmarks described earlier have been identified, we proceed with bone removal. Several methods have been described to find the IAC, but none are fail-safe, and the surgeon must make allowances for individual anatomy. According to the original description of this approach, the GSPN is followed posteriorly from the facial hiatus to the geniculate ganglion. Dissection then proceeds along the labyrinthine segment of the facial nerve to the IAC. ${ }^{6}$ This approach obviously places the facial nerve at risk because it is identified first and followed to the IAC.

A second method uses the SSC as the major landmark to localize the IAC. After drilling away the arcuate eminence, the underlying dense bone of the SSC is seen. As described earlier, the IAC lies in a $60^{\circ}$ plane from the ampulla of the SSC. This technique places the SSC, and therefore hearing, at risk. Orientation when using this approach may be aided by removal of the tegmen tympani and identification of the head of the malleus in the middle ear; the head of the malleus lies in the same plane as the geniculate ganglion and the IAC.

The final method depends on knowledge of the angles between the GSPN, the SSC, and the IAC.,3,10,13 The angle between the arcuate eminence (that is, the SSC) and the GSPN is $120^{\circ}$, and the angle between the arcuate eminence and the IAC is $60^{\circ}$. Therefore, the IAC bisects the angle between the GSPN and the arcuate eminence. These angles are not always reliable, however, because there may be considerable variability among individuals.
We prefer exposing the IAC medially first by drilling the porus acusticus and then proceeding laterally. The absence of neurovascular structures at the porus makes this area relatively safe for bone removal. Once the dura of the IAC is seen, the exposure continues laterally toward the fundus. At the fundus, bone resection is limited anteriorly by the basal turn of the cochlea and posteriorly by the ampulla of the SSC and the vestibule. Injury to either structure results in hearing loss. Therefore, the dural exposure is narrowed as the dissection proceeds laterally. A small bone spicule at the IAC fundus known as the vertical crest (Bill bar) separates the facial nerve from the superior vestibular nerve. The vertical crest is an important landmark for identification of the cochlea. Drilling in the angle between the GSPN and the IAC can easily perforate the underlying cochlea. The dura mater of the IAC is exposed just laterally to the vertical crest and opened longitudinally along the IAC axis. The dural opening is made posteriorly first over the superior vestibular nerve to avoid injury to the facial nerve. Closure of the wound in-

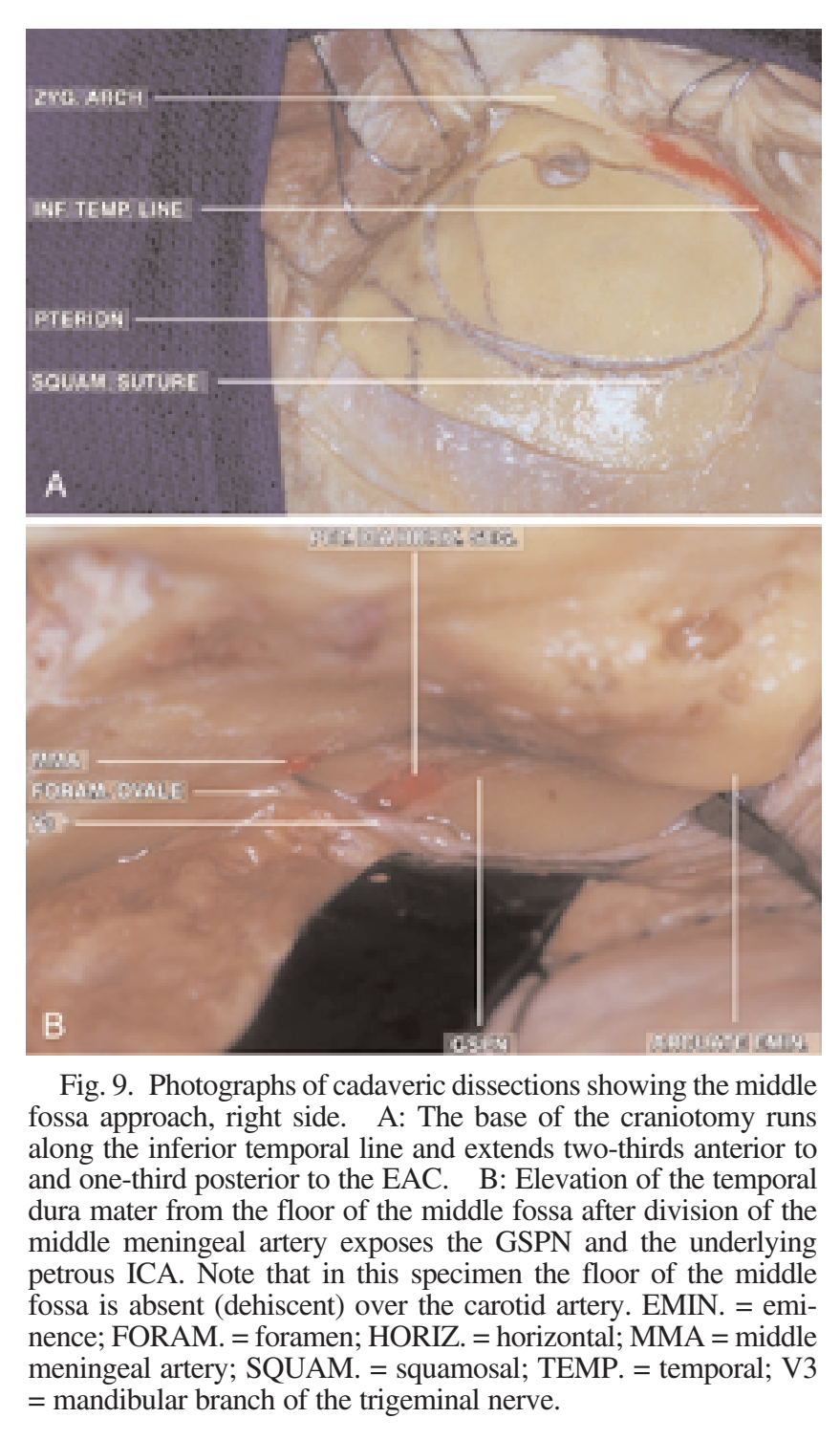


volves placement of a muscle or fat graft in the IAC. Any opening into the middle ear must also be obliterated to prevent a cerebrospinal fluid leak.

\section{Extended Middle Fossa Approach}

This dissection builds on the basic middle fossa approach with removal of additional medial petrous bone lying within the Kawase rhomboid. ${ }^{8}$ The anterior petrous bone is also resected to the horizontal segment of the petrous ICA; the Kawase rhomboid is bound by the GSPN laterally, the petrous ridge medially, the IAC axis posteriorly, and V3 anteriorly. The cochlea is at the posterolateral corner of the rhomboid. The shadow of the cochlea appears blue (blue line) as the overlying bone is gradually removed. The limits of the extended middle fossa exposure are the inferior petrosal sinus inferiorly, the middle ear ossicles laterally, the gasserian ganglion anteriorly, and the SSC posteriorly. The anterior petrosectomy exposes dura mater inferiorly to the level of the inferior petrosal sinus. The novice may be slightly disoriented at first with this approach, primarily because it is made via the middle fossa yet its goal is to expose posterior fossa dura. Opening the dura mater across the superior petrosal sinus exposes the posterior fossa. Lesions of the petrous apex, basilar trunk, and anterior brainstem may be treated using this approach.

\section{Kawase Approach}

This approach differs little from the extended middle fossa dissection. The extent of the anterior petrosectomy and dural opening is unchanged. The main addition is the transverse division of the tentorium to the incisura, which then allows full communication between the intradural middle and posterior fossa. ${ }^{9}$ The only two structures crossing the field of view are the fourth cranial nerve medially and the fifth nerve laterally. Elevating the divided edges of the tentorium with retention sutures provides wide exposure of the petroclival region, comfortably to midclivus, that is, level with the porus acusticus (Fig. 10). The enhanced view of the posterior fossa comes at the risk of injury to the trochlear nerve during the division of the tentorium. Although all of the middle fossa approaches are designed to preserve hearing, sacrifice of the labyrinth and thus hearing can sometimes be used to allow additional exposure of the posterior fossa. Figure 11 illustrates an ideal indication for the Kawase approach, in a case of dumbbell trigeminal schwannoma.

\section{CONCLUSIONS}

The transpetrosal approaches can be grouped into anterior and posterior categories. Each group is subclassified into individual approaches that represent a spectrum of bone resection and exposure of the posterior fossa. Both groups of approaches provide access to the CPA, the petroclival region, the BA, and the brainstem. The foundation for the posterior transpetrosal approach is the basic mastoidectomy. Increased degrees of bone resection anteriorly via the retrolabyrinthine, translabyrinthine, and transcochlear approaches provide graduated modules of exposure of the posterior fossa.

Similarly, the anterior transpetrosal approaches to the posterior fossa are a continuum of the basic middle fossa approach. The addition of an anterior petrosectomy and opening the temporal lobe dura mater enhances the view of the posterior fossa. Nevertheless, these approaches place the seventh cranial nerve between the surgeon and the posterior fossa, thus increasing the potential for facial palsy. These approaches also place the posterior fossa at the deepest part of the field, with the potential for difficult hemostasis.

In view of their anatomical characteristics, we believe that the retrolabyrinthine, translabyrinthine, and transcochlear approaches, when combined with a temporal craniotomy and tentorial sectioning, are best suited for middle and upper petroclival lesions located in the lateral, anterolater$\mathrm{al}$, and anterior brainstem, respectively. Similarly, anterior petrosectomies find their best applications in upper clival lesions, above the axial level of the clival midpoint, the porus.

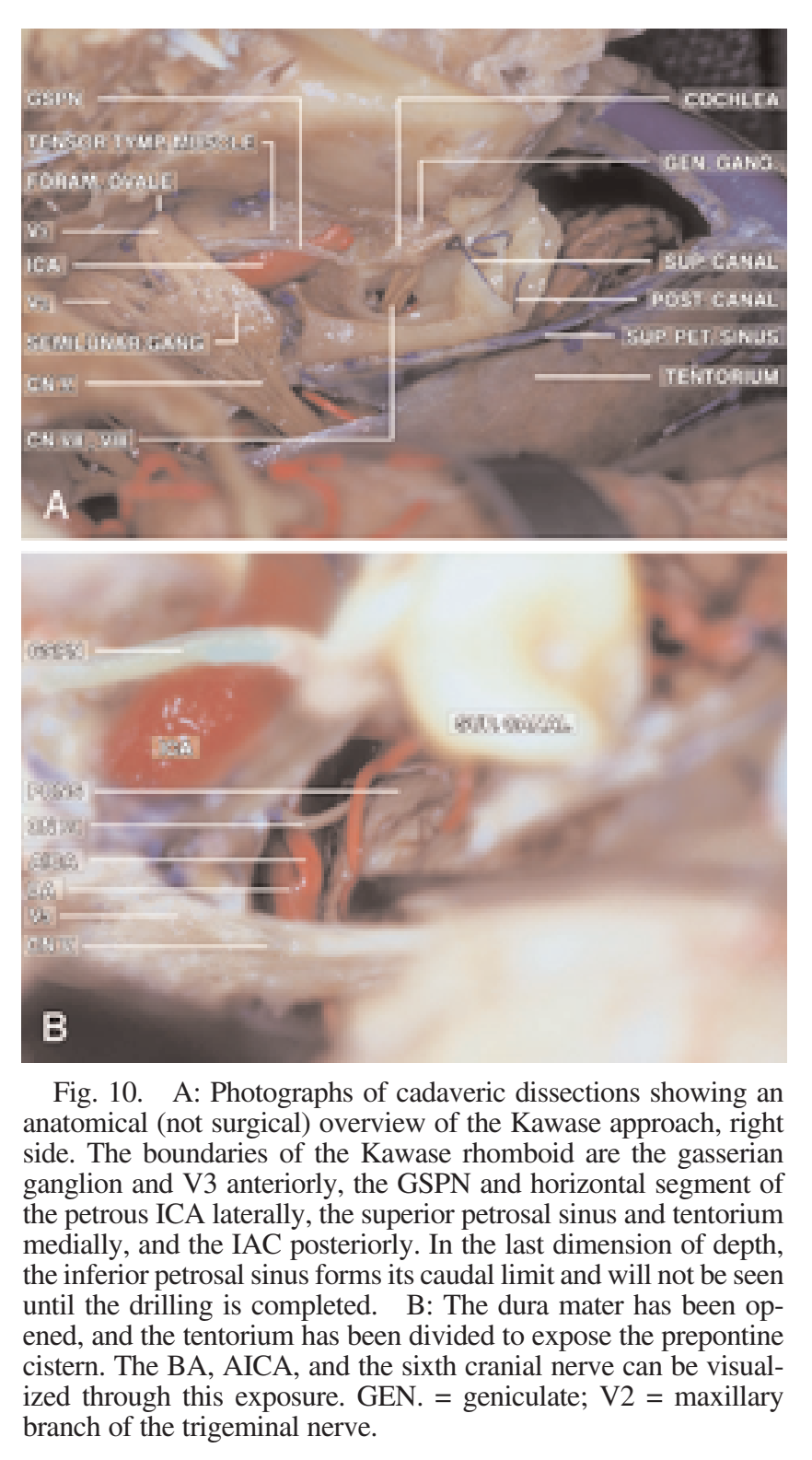



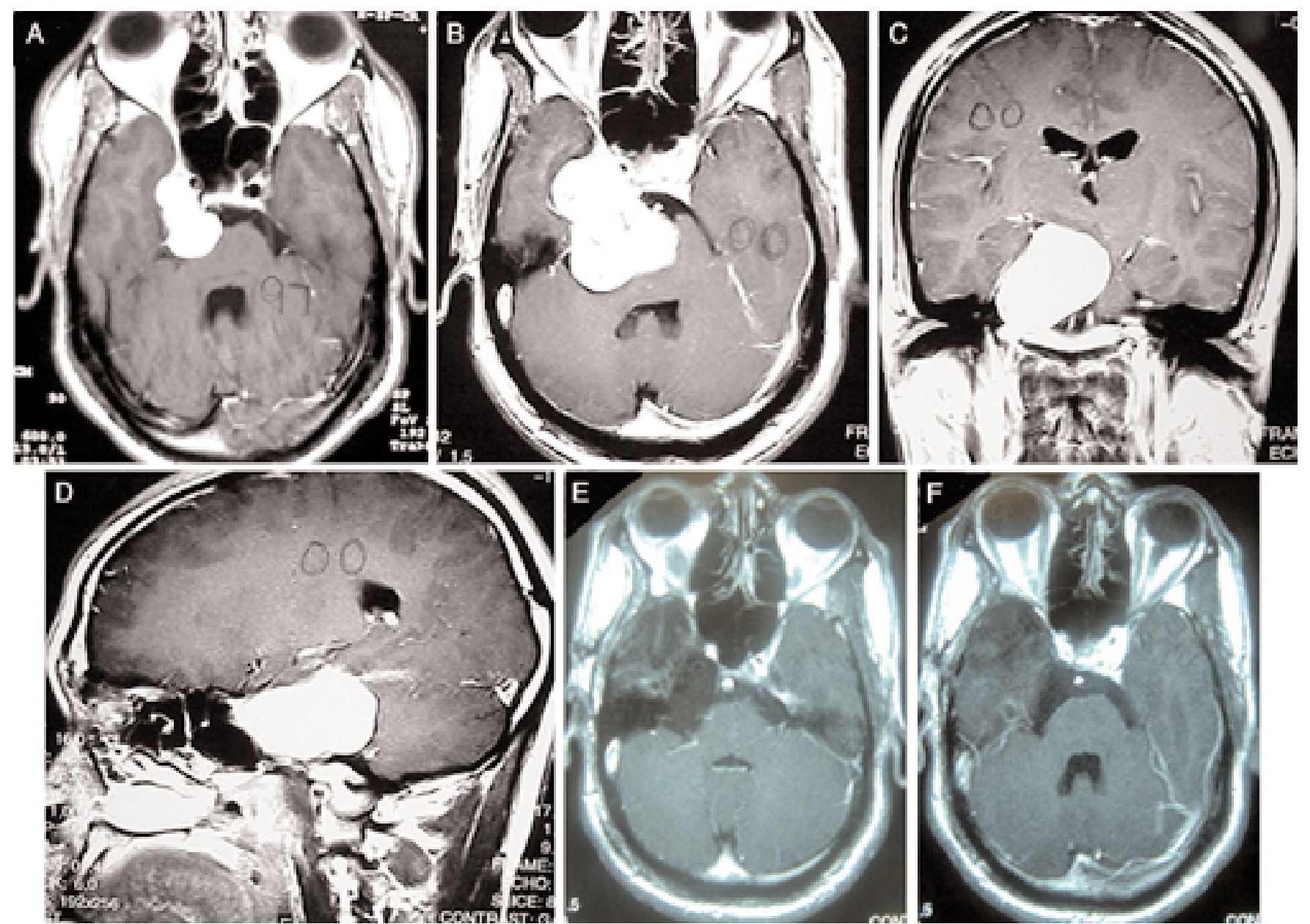

Fig. 11. This 38-year-old man presented originally at another facility with right-sided facial numbness. A: Contrast-enhanced $\mathrm{T}_{1}$-weighted axial MR image revealing a dumbbell-shaped mass with its center in the Meckel cave. The patient refused treatment and was lost to follow up. He presented to our institution 3 years later with worsening right face pain and weakness when chewing. B-D: Contrast-enhanced $\mathrm{T}_{1}$-weighted axial (B), coronal (C), and sagittal (D) images confirmed that the lesion had grown significantly. This schwannoma on the trigeminal nerve was resected completely via the Kawase approach. E and F: Images obtained 2 years postoperatively reveal no recurrence.

\section{References}

1. Bento RF, De Brito RV, Sanchez TG, et al: The transmastoid retrolabyrinthine approach in vestibular schwannoma surgery. Otolaryngol Head Neck Surg 127:437-441, 2002

2. Fisch U, Mattox D (eds): Microsurgery of the Skull Base. New York: Thieme, 1988, pp 436-457

3. Fisch U, Mattox D (eds): Microsurgery of the Skull Base. New York: Thieme, 1988, pp 546-576

4. Garcia-Ibanez E, Garcia-Ibanez JL: Middle fossa vestibular neurectomy: a report of 373 cases. Otolaryngol Head Neck Surg 88:486-490, 1980

5. House WF: Middle cranial fossa approach to the petrous pyramid. Report of 50 cases. Arch Otolaryngol 78:406-469, 1963

6. House WF, Hitselberger WE: The transcochlear approach to the skull base. Arch Otolaryngol 102:334-342, 1976

7. Kartush JM, Kemink JL, Graham MD: The arcuate eminence, topographic orientation in middle cranial fossa surgery. Ann Otol Rhinol Laryngol 94:25-28, 1985

8. Kawase T, Shiobara R, Toya S: Anterior transpetrosal-transtentorial approach for sphenopetroclival meningiomas: surgical method and results in 10 patients. Neurosurgery 28:869-876, 1991
9. Kawase T, Toya S, Shiobara R, et al: Transpetrosal approach for aneurysms of the lower basilar artery. J Neurosurg 63: 857-861, 1985

10. Lawton MT, Daspit CP, Spetzler RF: Transpetrosal and combination approaches to skull base lesions. Clin Neurosurg 43: 96-112, 1997

11. Miller CG, van Loveren HR, Keller JT, et al: Transpetrosal approach: surgical anatomy and technique. Neurosurgery 33: 461-469, 1993

12. Nelson RA: Temporal Bone Surgical Dissection Manual, ed 2. Los Angeles: House Ear Institute, 1991, pp 7-73

13. Rhoton AL Jr: The temporal bone and transtemporal approaches. Neurosurgery 47 (Suppl 1):S211-S264, 2000

Manuscript received July 11, 2005.

Accepted in final form July 18, 2005.

Address reprint requests to: Jacques J. Morcos, M.D., Department of Neurological Surgery, University of Miami, Lois Pope LIFE Center (2nd Floor), 1095 NW 14th Terrace (D4-6), Miami, Florida 33136. email: jmorcos@med.miami.edu. 